\title{
High density trans-admittance mammography development and preliminary phantom tests
}

Mingkang Zhao ${ }^{1}$, Hun Wi ${ }^{1}$, Abu Hena Mostofa Kamal ${ }^{1}$, Alistair Lee McEwan ${ }^{1,2}$, Eung Je Woo ${ }^{1}$ and Tong $\ln \mathrm{Oh}^{1 *}$

\footnotetext{
*Correspondence: tioh@khu.ac.kr 1 Department of Biomedical Engineering, Kyung Hee University, Yongin-si, Gyeonggi-do, 446-701, Korea

Full list of author information is available at the end of the article
}

\begin{abstract}
Background: Malignant breast tumor tissue has a significantly different electrical impedance spectrum than surrounding normal tissues. This has led to the development of impedance imaging as a supplementary or alternative method to $X$-ray mammography for screening and assessment of breast cancers. However low spatial resolution and poor signal to noise ratio has limited the clinical application.
\end{abstract}

Methods: In order to improve spatial resolution we developed a trans-admittance mammography (TAM) system including an array of $60 \times 60$ current sensing electrodes. We adopted a similar setup to X-ray mammography where the breast is situated between two holding plates. The top plate is a large solid metal electrode for applying a sinusoidal voltage over a range of frequencies from $50 \mathrm{~Hz}$ to $500 \mathrm{kHz}$. The bottom plate has 3600 current sensing electrodes that are kept at the ground potential. Currents are generated from the top voltage-applying electrode and spread throughout the breast, entering the TAM system through the array of current sensing electrodes on the bottom plate. The TAM system measures the exit currents through 6 switching modules connected to 600 electrodes each. Each switching module is connected to 12 ammeter channels which are switched sequentially to 50 of the 600 electrodes each measurement time. Each ammeter channel is comprised of a current-to-voltage converter, a gain amplifier, filters, an analog to digital converter, and a digital phase sensitive demodulator.

Results: We found an average noise level of $38 \mathrm{nA}$, amplitude stability of less than $0.2 \%$, crosstalk of better than $-60 \mathrm{~dB}$ and $70 \mathrm{~dB}$ signal to noise ratio over all channels and operating frequencies. Images were obtained in time difference and frequency difference modes in a saline phantom.

Conclusion: We describe the design, construction, and calibration of a high density TAM system in detail. Successful high resolution time and frequency difference images showed regions of interest with the expected admittivity changes in the frequency spectrum.

Keywords: Trans-admittance, Conductivity spectrum, Breast cancer, Lesion detection PACS MCS codes: Admittance measurement, 84.37. + $q$ Mammography in computer-aided diagnosis, 87.57.rh Electric impedance measurement, 84.37. $+q$ 


\section{Background}

Experimental findings show that cancerous breast tissues have significantly higher admittivity values compared with those of normal breast tissues [1-4]. For the diagnosis of breast cancer, several bioimpedance techniques have been suggested as an alternative or supplementary method to X-ray mammography. The urgent need here is to assist in reducing the large number of biopsies resulting from mammography. To apply the bioimpedance technique for breast imaging, two different approaches of tomographic and projection imaging have been proposed.

Electrical impedance tomography (EIT) is a tomographic imaging method to visualize an admittivity distribution inside the human body [5,6]. EIT typically uses a ring of electrodes placed around the body of interest. Breast imaging using EIT was proposed by Larson-Wiseman (1998), Mueller et al (1999), Cherepenin et al (2001 and 2002), Kerner et al (2002) and Kao et al (2003) [7-12]. While previous systems sought to allocate rings of electrodes around the breast, the latest research efforts are focused on imaging the breast using a fixed planar array of electrodes (projection imaging) as imaging is highly sensitive to relative electrode positions. The idea of using a handheld probe with a planar array of electrodes was first suggested by Kao et al (2006) who used two radiolucent plates of planar electrode arrays with the breast situated between them [13,14]. This design allows a dual-mode operation of EIT and X-ray mammography in the X-ray mammography geometry. Based on a three-dimensional forward model of the mammography geometry, Choi et al (2007) proposed a linear conductivity image reconstruction algorithm with a regularization method [15]. For the same geometry, Boverman et al (2008) developed a linear admittivity image reconstruction algorithm for spectroscopic imaging in the range of $3 \mathrm{kHz}$ and $1 \mathrm{MHz}$ [16]. Kao et al (2008) reported promising results of their preliminary patient study by relating regional admittivity spectra registered to 3D mammograms [17]. More patient studies with improved hardware, software and algorithms are expected to validate the ability to differentiate benign and malignant lesions, and establish the clinical significance of impedance imaging in the diagnosis of breast cancer.

In 1978, Henderson and Webster suggested a frontal plane impedance camera, which is considered to be the first medical impedance imaging method. Applying a constant voltage on the frontal plane of the chest using a large electrode, current enters and spreads throughout the chest. By attaching an array of reference electrodes on the back and measuring the exit current through each one of them, we may draw a two-dimensional map of the current distribution, which can be interpreted as a projection image of the admittivity distribution. This frontal plane impedance camera initiated medical impedance imaging research, however it received much less attention than the tomographic impedance imaging method until a commercial system called T-Scan was introduced for adjunctive clinical uses with X-ray mammography $[18,19]$. In the T-Scan configuration, a patient holds a voltage electrode with one hand and a flat scan probe is placed on her breast. The probe includes an array of electrodes at zero volts. Measured exit currents from all electrodes of the scan probe provided a two-dimensional trans-admittance image, visualising the admittivity distribution inside the local breast region underneath the scan probe.

The diagnostic information from the T-Scan system lacks a sophisticated reconstruction method of lesion localization, hence Seo et al (2004) and Ammari et al (2004) developed a mathematical framework to analyze the trans-admittance map and suggested a non-iterative algorithm to extract core features of lesions from two maps with and 
without a lesion [20,21]. Noting that admittivity spectra of cancerous and normal breast tissues are different, Oh et al (2007) and Kim et al (2008) suggested a frequency-difference method and showed its feasibility from numerical simulations and phantom experiments $[22,23]$. For their experimental study, Oh et al (2007) developed a multi-frequency trans-admittance scanner (TAS) and a cylindrical scan probe with 320 current-sensing electrodes [22]. Limited by the radius of the scan probe, its field of view was restricted to $20 \mathrm{~mm}$ and the spatial resolution of the trans-admittance maps was also limited by the relative small number of current-sensing electrodes.

In this paper, we describe a new design of the trans-admittance scanner for the mammography setup (TAM) with better spatial resolution. Instead of using the handheld voltage electrode and scan probe, we use two plates with the breast between them. The imaging setup is identical to X-ray mammography except that we may rotate the two plates for consecutive projection imaging at any angle. After describing its basic performance including the signal-to-noise ratio (SNR), stability, noise and crosstalk between measurement channels, we will show time-difference and frequency-difference TAM images of a breast phantom with a known admittivity distribution. We hypothesize that the regions of interest within the frequency-difference TAM images reflect the expected admittivity changes in the frequency spectrum.

\section{Methods}

\section{System architecture}

Figure 1 shows the measurement configuration and the structure of the TAM system. The breast is placed between two plates. We apply a constant sinusoidal voltage over a range of frequencies from $50 \mathrm{~Hz}$ to $500 \mathrm{kHz}$ to the large top plate of $180 \times 180 \mathrm{~mm}$ stainless steel. The bottom plate consists of 3600 gold plated current sensing electrodes situated on

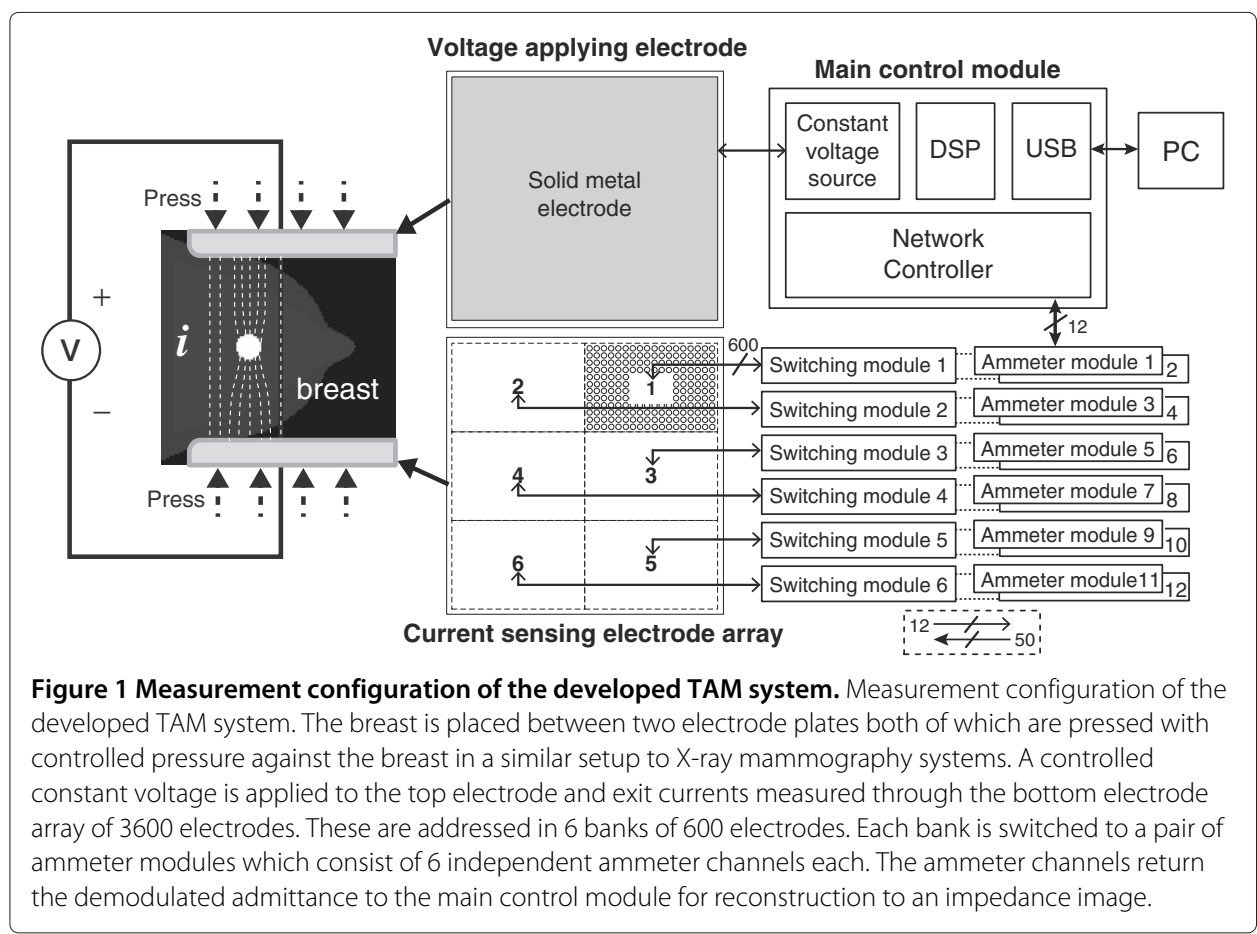


a PCB. These are kept at the ground potential. From the potential difference between the two plates, current spreads throughout the breast and returns to the current sensing electrode array. Any conductivity anomaly distorts the current flow and its effect is mapped on the plane of current sensing electrodes. The measured exit current is varied by the trans-admittance distribution which is sensitive to the presence of lesions that create conductivity anomalies in the homogenous breast tissue. The exit current measurements can be reconstructed as a TAM image when considering the applied voltage. The two parallel electrode plates can be rotated around the breast to increase the measurement depth. The spatial resolution of measured trans-admittance maps is limited by the number and size of the current sensing electrodes. We chose the size of current sensing electrode array large enough to cover the whole breast when it is pressed. The TAM system comprises one voltage applying electrode, 3600 current sensing electrodes, 6 switching modules, 12 ammeter modules, a constant voltage source and a main control module including a network controller for communication between a PC and ammeter controllers as shown in Figure 1. Each functional block will be explained in following sections.

\section{Current sensing electrodes and switching module}

The 3600 current sensing electrode array is connected to 6 switching modules each addressing 600 adjacent electrodes. Each current sensing electrode is a gold-coated, $2 \mathrm{~mm}$ diameter, circular contact on a printed circuit board (PCB) with the commonly used FireRetardant glass laminate substrate (FR4). The spacing between adjacent electrodes is $3 \mathrm{~mm}$ center-to-center, leaving $1 \mathrm{~mm}$ gaps. On the back side of this PCB, we placed 18 surface mount connectors (MEG-array mezzanine connectors, FCI, France) for 3600 signal paths. Each connecter has 200 pins and it connects between each current sensing electrode and the corresponding input of a switch in one of the switching modules. At each measurement time, 12 signal outputs among the 600 electrodes are connected to 12 independent ammeter channels. This is repeated 50 times to cover all 600 electrodes. The 12 ammeter channels belong to one of the 2 ammeter modules shown in Figure 1 so that each ammeter module contains 6 ammeter channels. This arrangement was chosen to enable a compact and flexible design. Figure 2 shows the functional blocks for a switching module connected to 2 ammeter modules or 12 ammeter channels. The electrode output is switched either to the circuit ground or to the input of a chosen trans-resistance amplifier in the ammeter channel.

\section{Ammeter}

Two ammeter modules are responsible for 600 current sensing electrode outputs. One ammeter module consists of 6 ammeter channels, one of which is highlighted in Figure 3. The remaining 3 channels are located on the backside of this PCB. The exit current from a selected electrode is connected to the input of an ammeter channel. The front-end of each ammeter channel is a trans-resistance amplifier that converts the exit current from a selected electrode to the amplified voltage signal. The current to voltage (I-V) converter provides a virtual ground to the input for measuring the exit current [24]. We amplify the output of the I-V converter using a variable gain voltage amplifier and filter out the noise signal. A high speed analog to digital converter (ADC) quantizes the amplified and filtered signal for digital demodulation. The input dynamic range of the ammeter is $\pm 2.5 \mathrm{~mA}$ with minimum gain and the amplitude resolution is $3 \mathrm{nA}$ with the maximum gain setting. 


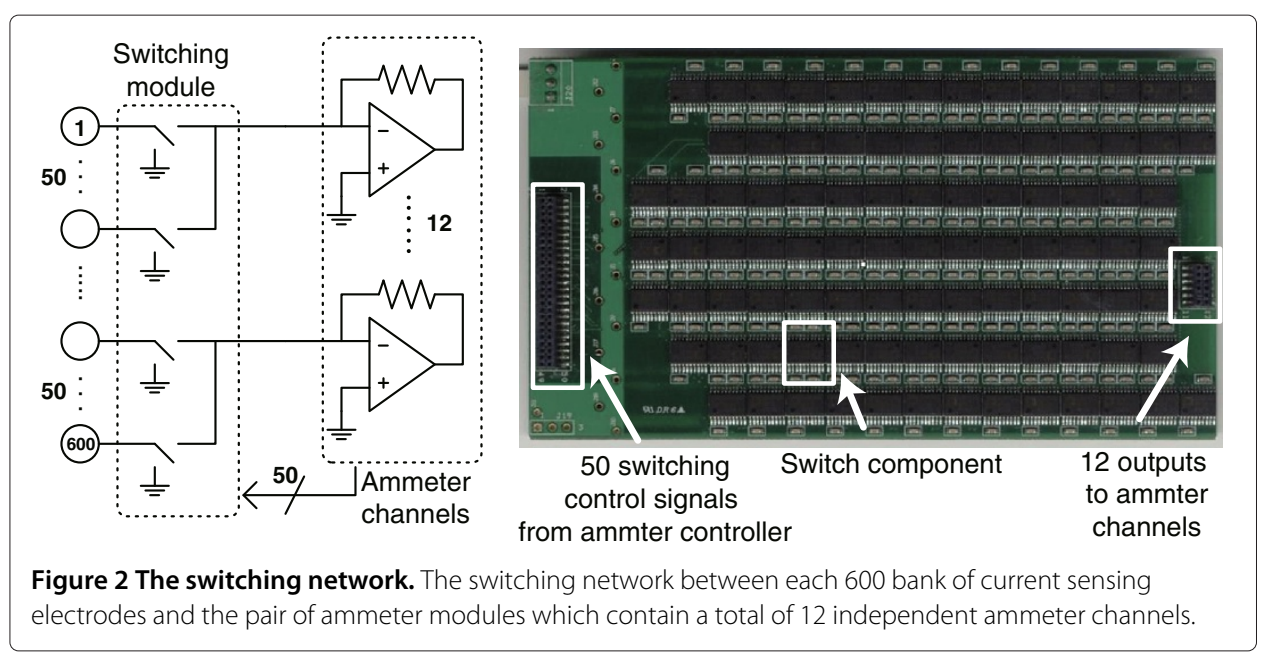

An ammeter controller for each ammeter module is implemented on a field programmable gate array (FPGA) (EP3C10F256C8N, Altera, USA) including 6 independently operating digital phase sensitive demodulators to calculate real and imaginary components of measured signals in parallel, and other control logic. The demodulator outputs are transferred to a PC for presenting images.

One ammeter controller is the master which is responsible for the generation of the 50 switching control signals that change the switch configuration in synchrony with the data acquisition time. The other ammeter controller is the slave and does not generate switching control signals. One switching control signal is shared by 12 switches. The ammeter controller operates multiple ADCs synchronously and controls gains for the amplifiers.

\section{Main control module with constant voltage source}

The main control module consists of a constant voltage source, a main controller implemented with a DSP (TMS320F2812, Texas Instruments, USA), an intra-network controller, and an isolated USB interface for communicating with a PC as shown in

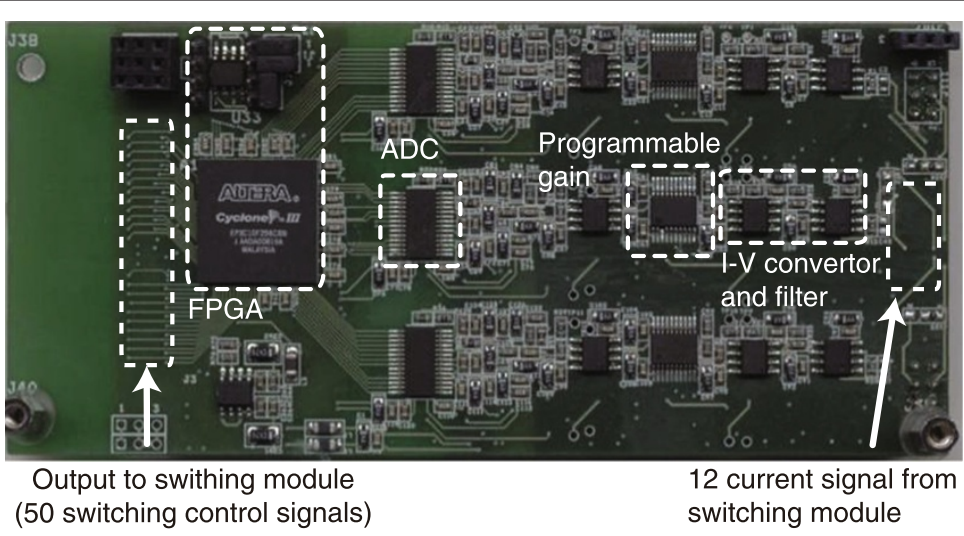

Figure 3 Ammeter module. Ammeter module with one ammeter channel highlighted. Each side of the PCB contains 3 ammeter channels. These consist of a trans-resistance amplifier (I-V converter), filter, programmable gain amplifier and ADC. Each ammeter channel sends the digitized current to the single FPGA for independent demodulation. 
Figure 4. The intra-network controller and digital waveform generator are implemented on a FPGA. The intra-network controller arbitrates commands and data between the main controller and each of the ammeter controllers through multiple serial ports in a star configuration. The constant voltage source is implemented with a recent digital to analog converter (DAC) (AD9783, Analog Devices, USA) and operational amplifier. The DAC component includes two 16-bit DACs for generating sinusoid waveforms and two additional 10-bit DACs for compensating output offset and range controls. Analog outputs are summed and fed to a power amplifier (AD8018, Analog Devices, USA) capable of driving low distortion signals. We inserted a series current sensing resistor, $100 \Omega$, at the voltage output. A voltmeter in the main control module measures the voltage difference across this series resistor to estimate the total amount of current injected into the tested object. A digital waveform generator controls the total amount of injected current below the safety level and records the magnitude and phase for compensating the measured data. The timing control module operates with the digital waveform generator to produce control signals for the switching module. The main controller manages the entire measurement process and communicates with a PC by a customized isolated USB connection consisting of a USB controller (C8051F320, Silicon Laboratory, USA) and digital signal isolator (ADuM4160, Analog Devices, USA).

\section{Calibration}

The ground potential and virtual ground of the I-V converter input are not identical. The input resistances and capacitances from PCB routes, wires, connectors and switches also produce inconsistencies in different electrode connections. We remove such systematic artifacts due to non-ideal characteristics of the TAM system using following calibration method.

Calibration is performed using a rectangular saline tank where the top and bottom electrodes tightly fit the enclosure to prevent leakages. We calibrated TAM system with saline phantom in order to include the half cell potential and impedance characteristics of the electrodes. The calibration will not depend on the saline conductivity as long as the saline is within the measurable impedance range of the system. Inside the homogeneous saline tank, current density is uniform and parallel to the $z$-direction. First, we calculate

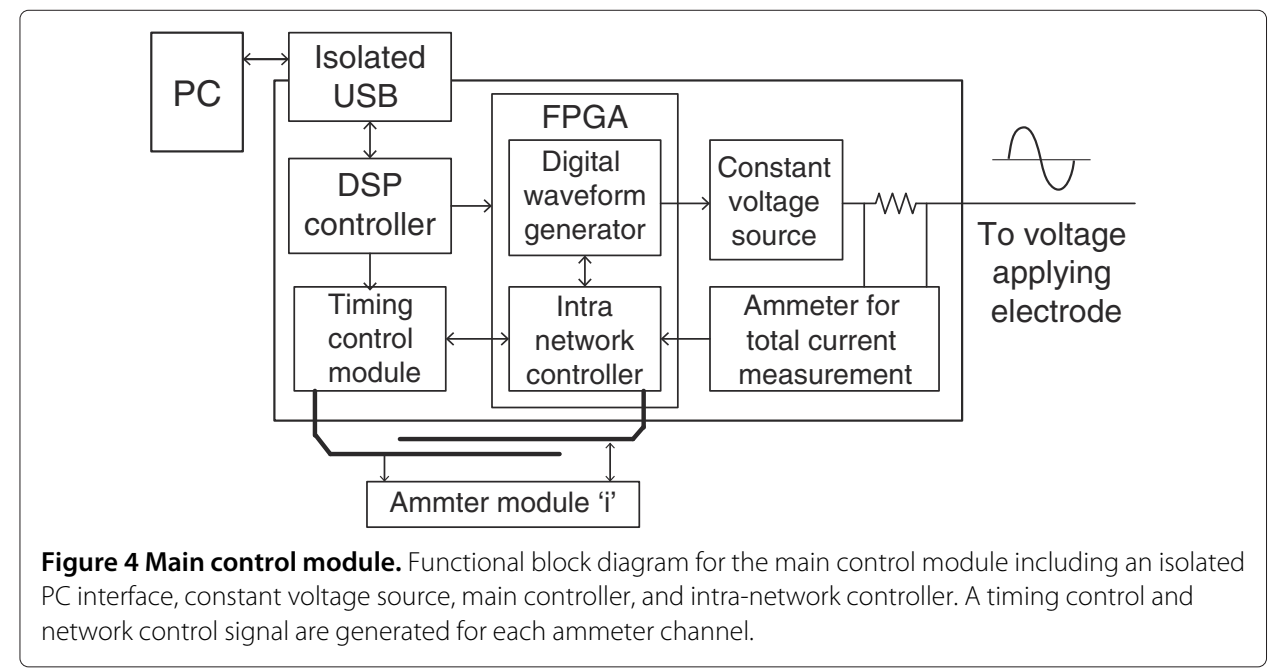


phase compensation factors for each electrode to reduce the phase to zero, as expected for a purely resistive load. After applying phase compensation, we compute the averaged measurements of exit current from all current sensing electrodes in all possible switch combinations and find the magnitude scaling factors that make the exit current values equal to the averaged reference measurement value using fractional deviations. We store calibration factors and measured applied currents for each frequency because the amount of applied current is different depending on the operating frequency. This allows us to compensate the trans-admittance map with the measured amount of applied current and calibration factors at the specific operating frequency.

\section{Trans-admittance maps}

The breast is situated between the two electrode plates as shown in Figure 1. To develop the trans-admittance map we consider the three-dimensional domain $\Omega$ of the breast bounded by its surface $\partial \Omega$. We apply a constant voltage of $A$ volts with an angular frequency $\omega$ to the large electrode plate with contact area $\Gamma_{S}$. The other electrode plate contains the 3600 current sensing electrodes at ground potential. These form a total contact area of $\Gamma_{M}$. The electric field between the plates can be expressed as a complex voltage $V(\mathbf{r})$ at position $\mathbf{r}=(x, y, z)$ in $\Omega$. This satisfies the following mixed boundary value problem:

$$
\left\{\begin{array}{l}
\nabla \cdot((\sigma+i \omega \epsilon) \nabla V(\mathbf{r}))=0, \quad \mathbf{r} \in \Omega \\
V(\mathbf{r})=0, \quad \mathbf{r} \in \Gamma_{M} \\
V(\mathbf{r})=A, \quad \mathbf{r} \in \Gamma_{S} \\
(\sigma+i \omega \epsilon) \nabla V(\mathbf{r}) \cdot \mathbf{n}(\mathbf{r})=0, \quad \mathbf{r} \in \partial \Omega \backslash\left(\Gamma_{S} \cup \Gamma_{M}\right)
\end{array}\right.
$$

where $\mathbf{n}$ is the unit outward normal vector to the boundary, $\sigma=\sigma(\mathbf{r}, \omega)$ the conductivity and $\epsilon=\epsilon(\mathbf{r}, \omega)$ the permittivity. Both $\sigma$ and $\epsilon$ depend on $\mathbf{r}$ and $\omega$. Denoting the real and imaginary parts of $V$ by $v=\Re V$ and $h=\Im V$, we have the following coupled system:

$$
\left\{\begin{array}{cl}
\nabla \cdot(\sigma \nabla v)-\nabla \cdot(\omega \epsilon \nabla h)=0 & \text { in } \Omega \\
\nabla \cdot(\omega \epsilon \nabla v)+\nabla \cdot(\sigma \nabla h)=0 & \text { in } \Omega \\
v=0 \text { and } h=0 & \text { on } \Gamma_{M} \\
v=A \text { and } h=0 & \text { on } \Gamma_{S} \\
\mathbf{n} \cdot \nabla v=0 \text { and } \mathbf{n} \cdot \nabla h=0 & \text { on } \partial \backslash \backslash\left(\Gamma_{S} \cup \Gamma_{M}\right) .
\end{array}\right.
$$

In order to detect a lesion underneath the current sensing electrodes, we measure the distribution of exit currents through $\Gamma_{M}$ as

$$
-g(\mathbf{r}):=\underbrace{\mathbf{n} \cdot(\sigma \nabla v-\omega \epsilon \nabla h)}_{\text {real part }}+i \underbrace{\mathbf{n} \cdot(\sigma \nabla h+\omega \epsilon \nabla v)}_{\text {imaginary part }}, \quad \mathbf{r} \in \Gamma_{M} .
$$

The distribution of exit currents $g$ is called the trans-admittance map when an applying voltage is constant.

We suppose that there is a cancerous lesion $D$ inside $\Omega$ as shown in Figure 1. The complex conductivity $\tau:=(\sigma+i \omega \epsilon)$ changes abruptly across the interface $\partial D$ and we assume

$$
\tau:=\sigma+i \omega \epsilon= \begin{cases}\sigma^{n}+i \omega \epsilon^{n} & \text { in the normal tissue } \Omega \backslash \bar{D} \\ \sigma^{c}+i \omega \epsilon^{c} & \text { in the cancerous lesion } D .\end{cases}
$$

Both trans-admittance maps $g_{1}$ and $g_{2}$ are simultaneously acquired at two different frequencies $\omega_{1}$ and $\omega_{2}$, respectively, from the breast region $\Omega$ with a lesion $D$ [22]. 


\section{Multi-frequency saline phantom experiment}

The developed TAM system, shown in Figure 5, was evaluated in terms of noise, SNR, the waveform stability error of the constant voltage source, and the crosstalk between measurement channels. We first measured the noise levels of the ammeters by filling the gap between the top and bottom plates with $0.2 \mathrm{~S} / \mathrm{m}$ saline. All measured deviations in exit currents from each electrode are considered noise. We measured the percentile stability error of the constant voltage source directly on the current sensing resistor, at multiple operating frequencies, and without using the phase-sensitive demodulator and I-V converter. Crosstalk was assessed between switches or ammeter channels. Finally, we measured the overall performance of the TAM system as SNR defined by the ratio of the mean value to the standard deviation of repeated measurements using the saline tank. We measured the real and imaginary components of exit currents to compute the SNR.

In order to show the imaging performance of TAM system at multiple frequencies, we produce trans-admittance maps with objects which have different admittivity spectra and are similar to biological tissues. As the reference, we fill the $15 \mathrm{~cm}$ deep phantom with $0.2 \mathrm{~S} / \mathrm{m}$ saline. We inserted an agarose cuboid of $43 \times 30 \times 20 \mathrm{~mm}^{3}$ in the left side positioned at $(-45 \mathrm{~mm},-9 \mathrm{~mm},-19 \mathrm{~mm})$ while a cylindrical carrot with $35 \mathrm{~mm}$ diameter and $20 \mathrm{~mm}$ height is positioned on the right side $(45 \mathrm{~mm},-9 \mathrm{~mm},-19 \mathrm{~mm})$ as shown in Figure 6(a). The top surfaces of both objects are $9 \mathrm{~mm}$ away from the current sensing electrode array. We obtain the current distribution data at 10 different frequencies, from $50 \mathrm{~Hz}$ to $500 \mathrm{kHz}$ with a constant voltage of $1.4 \mathrm{~V}$. Using a simple frequency difference algorithm we reconstruct frequency difference images from the measured trans-admittance maps obtained at different frequencies [25,26]. The admittance values from cross-sections of simulated maps of our phantom at different electrode spacing are shown in Figure 6(c). These demonstrate that there is improvement in the spatial resolution when decreasing the spacing of electrodes from $18 \mathrm{~mm}$ to $3 \mathrm{~mm}$. In our test phantom geometry this corresponds to an increase from 600 to 3600 electrodes, thus providing motivation to increase the number of electrodes over previous TAM designs.

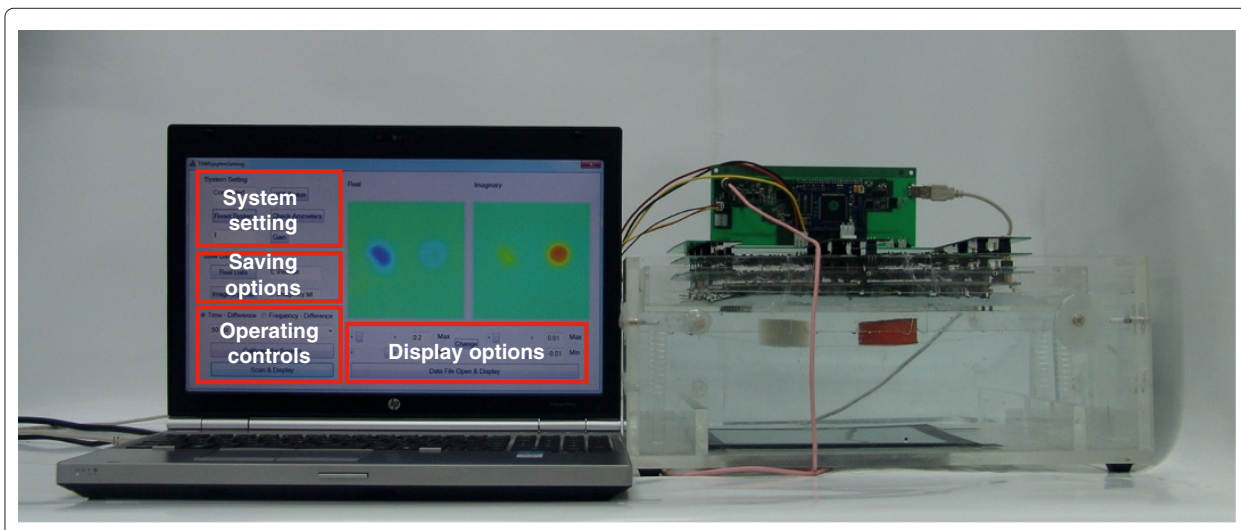

Figure 5 The developed TAM system with user interface and saline phantom. The developed TAM system with user interface software and saline phantom. The electrodes shown here are inverted with the large single voltage applying electrode plate on the bottom of the rectangular perspex tank. The array of current sensing electrodes are on the top surface of the tank to move them away from possible leaks or spillages. 
Figure 6 Phantom setup and simulation results. (a) Conductivity saline phantom with 2 anomalies (cuboid agar and cylindrical carrot), (b) conductivity spectrum for each testing material, (c) admittance of the cross-section over the center of the testing objects for various levels of electrode spacing. The total size of the phantom was constrained so an increased spacing corresponds to a simulation of less electrodes. i.e. the spacing of $3 \mathrm{~mm}, 9 \mathrm{~mm}$ and $18 \mathrm{~mm}$ correspond to 3600,1200 and 600 electrodes. (a)

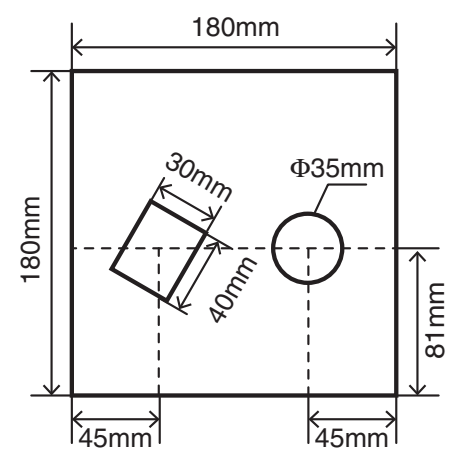

(b)

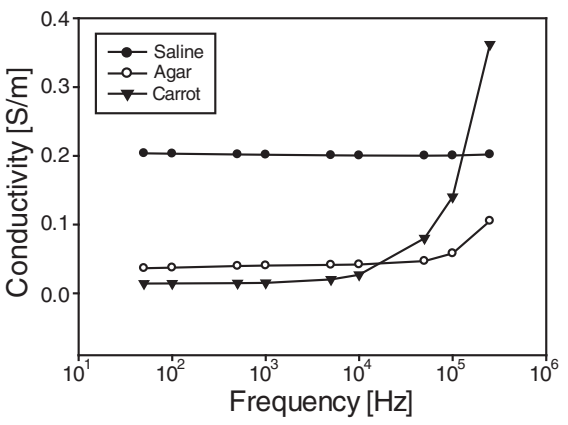

(c)

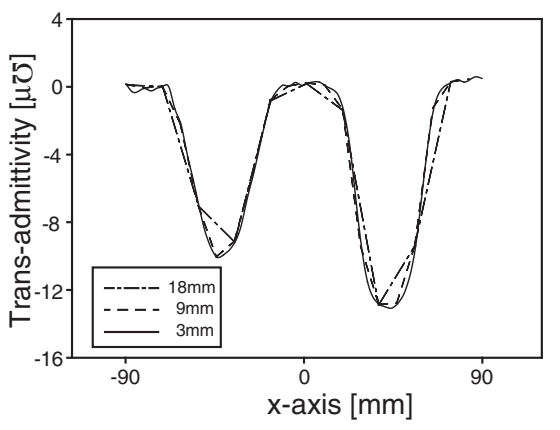

\section{Results}

\section{Basic performance}

Figure 7(a) shows the noise amplitudes with saline between the two electrode plates at each operating frequency. The average magnitude of noise within the operating range was $38 \mathrm{nA}$. Noise was less than $5 \mathrm{nA}$ for frequencies greater than $1 \mathrm{kHz}$. The amplitude stability error of the constant voltage source was $0.13 \pm 0.03 \%$ at all operating frequencies as shown in Figure 7(b). The crosstalk between neighboring channels on the switches, ammeter channels, and ammeter modules is shown in Figure 7(c). All operating frequencies produced SNR of better than $70 \mathrm{~dB}$.

\section{Time-difference TAM images}

Figures 8(a) and 9(a) show the time-difference real- and imaginary-part images at 10 different frequencies. Figures 8(b), 8(c), 9(b) and 9(c) show the central admittance cross 
(a)

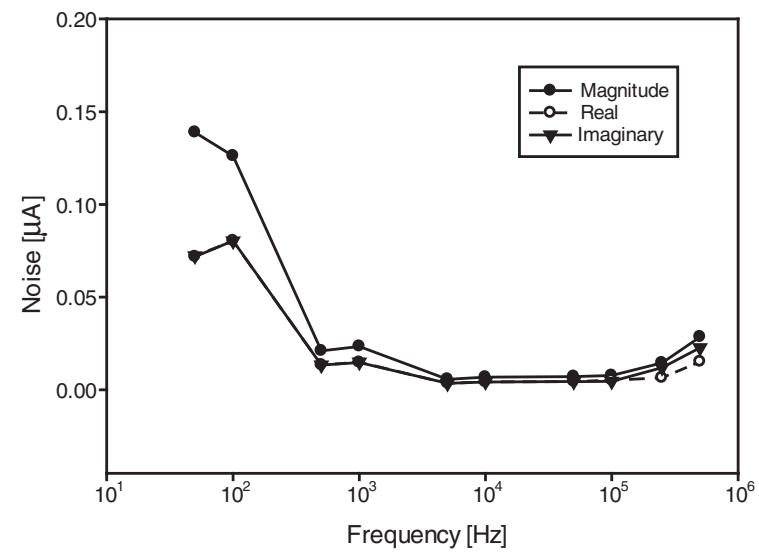

(b)

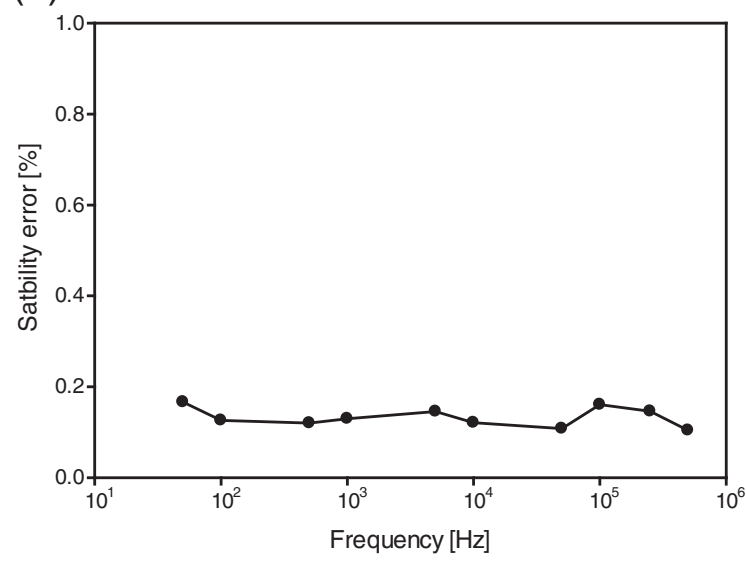

(c)

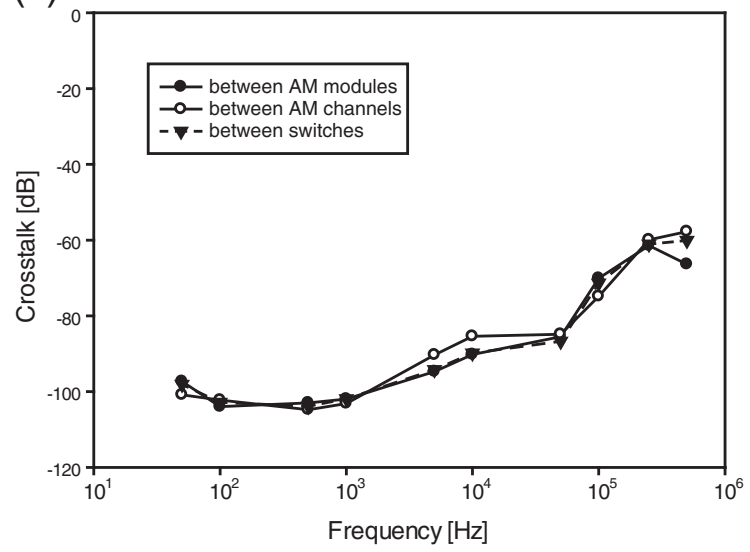

Figure 7 Measurement of noise, amplitude stability and crosstalk. (a) Noise spectra of magnitude, real, and imaginary components of measured exit currents, and (b) amplitude stability error of the constant voltage source. (c) Crosstalk between ammeter modules, adjacent ammeter channels, and neighboring switches in the switching module. 


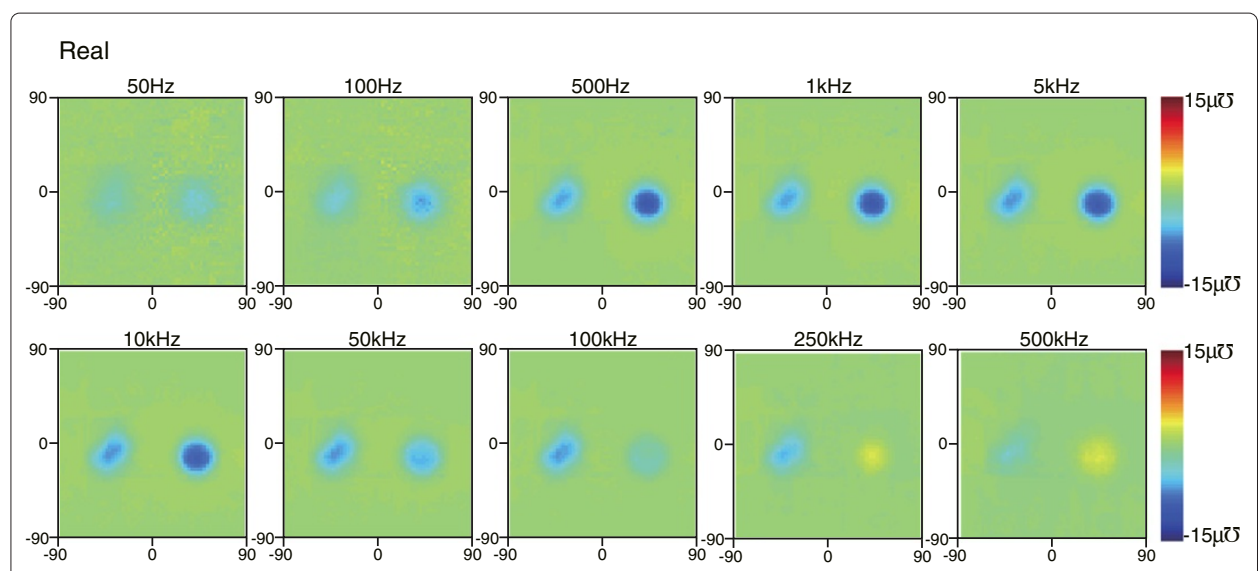

(a)

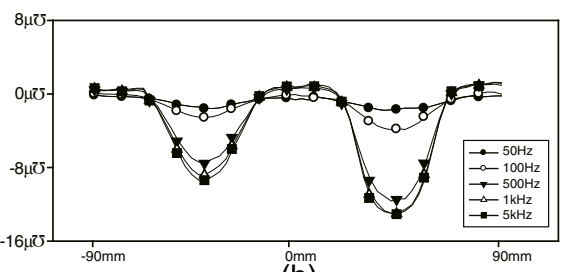

(b)

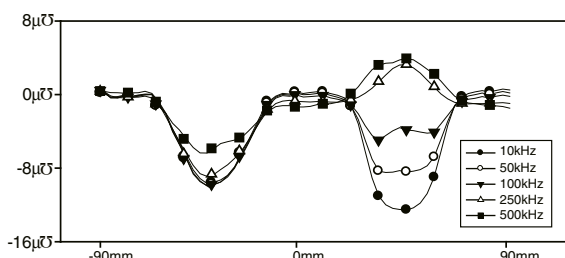

(c)

Figure 8 Time difference images of the real component of admittance. (a) Time difference real trans-admittance maps for the testing objects obtained at 10 different frequencies. The images are displayed for the full $180 \mathrm{~mm} \times 180 \mathrm{~mm}$ size of the electrode array using a RGB colorbar between $-15 \mathrm{u} \mho \mathrm{and}+15 \mathrm{u} \mho$ as is customary in admittivity images. (b) and (c) Admittance of the cross section over the center of the testing objects at each frequency.

section of the objects in each image. We measured reference conductivity of background saline, agar, and carrot at the operating frequencies of the TAM system using an impedance analyzer (1260A, AMETEK Inc., UK) as shown in Figure 7(b).

\section{Frequency-difference TAM images}

Figure 10 shows the magnitude and phase images of the trans-admittance distribution obtained at 6 different frequency pairs with respect to the $500 \mathrm{~Hz}$.

\section{Discussion}

\section{Basic performance}

We developed a high density TAM system capable of producing trans-admittance projection images of conductive objects. We confirmed the performance of the TAM system with noise spectrum, amplitude stability of the constant voltage source, crosstalk on measurement channels, and SNRs within the operating frequency range of $50 \mathrm{~Hz}-500 \mathrm{kHz}$. As shown in Figure 7 the system noise increased at the lower frequencies because the measurement time is related to the period of the operating frequency. The measurement time reduces with higher frequencies, example times are $64 \mathrm{~s}$ for $50 \mathrm{~Hz}, 1.6 \mathrm{~s}$ for $5 \mathrm{kHz}$ and $12.8 \mathrm{~ms}$ for $500 \mathrm{kHz}$. These are not proportional due to our use of discrete clock division and the overhead of additional data transfers and synchronization wait times explained in a previous paper on an EIT system which forms the base architecture of the TAM system [25]. We used a filter to remove low frequency biosignals and DC components. The signal below $100 \mathrm{~Hz}$ is affected by this filter and drifts in conductivity and temperature. This 


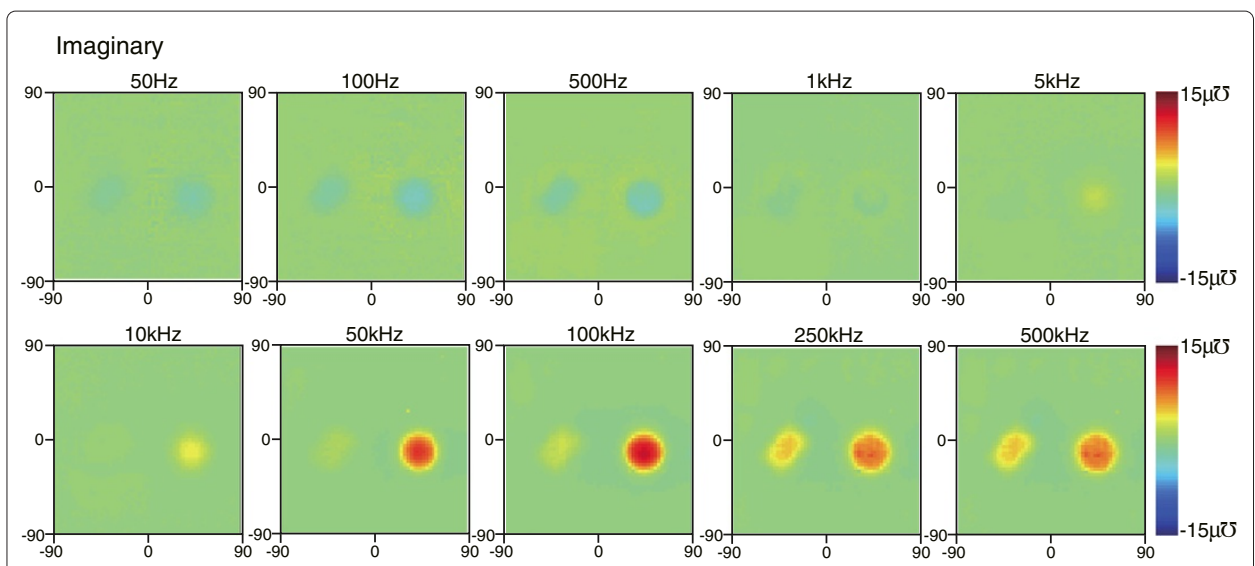

(a)

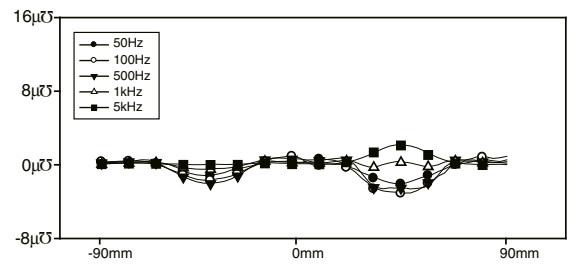

(b)

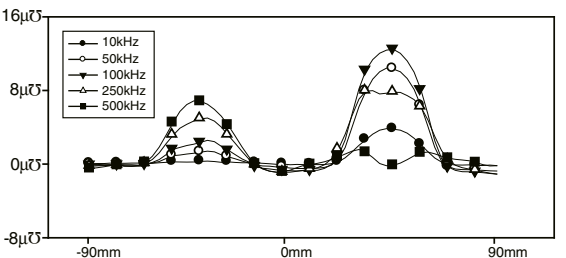

(c)

Figure 9 Time difference images of the imaginary component of admittance. (a) Time difference imaginary trans-admittance maps for the testing objects obtained at 10 different frequencies. The images are displayed for the full $180 \mathrm{~mm} \times 180 \mathrm{~mm}$ size of the electrode array using a RGB colorbar between $-15 \mathrm{u} \mho$ and $+15 \mathrm{u} \mho$ as is customary in admittivity images. (b) and (c) Admittance of the cross section over the center of the testing objects at each frequency.

was evident as we also saw the SNR of low frequencies degrading when the measurement time was increased.

The TAM system has a complex configuration with many current sensing electrodes and switches for measuring data with a small number of measurement channels. We examined crosstalk between neighboring channels on the switches, ammeter channels, and ammeter modules. Figure 7 (c) shows that the average crosstalk was degraded from $-98.5 \mathrm{~dB}$ below $10 \mathrm{kHz}$ to $-60 \mathrm{~dB}$ at $500 \mathrm{kHz}$. We examined crosstalk between switch input ports, adjacent ammeter channels, and ammeter channels belonging to different

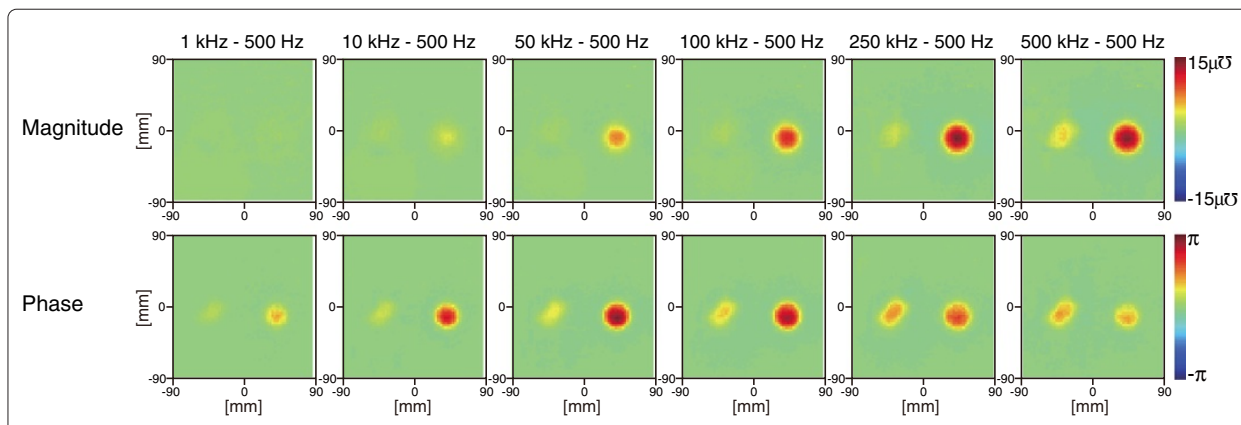

Figure 10 Frequency difference images. (a) Frequency difference images between 6 frequency pairs. The images are displayed for the full $180 \mathrm{~mm} \times 180 \mathrm{~mm}$ size of the electrode array using a RGB colorbar between $-15 u \mho$ and $+15 u \mho$ as is customary in admittivity images. Here we have displayed magnitude and phase images as they more clearly show the conductivity magnitude and phase change over frequency. 
ammeter modules. They produced a similar frequency spectrum as shown in Figure 7(c). Most of the crosstalk was induced by the switching modules and little was added by the measurement routes on ammeter channels and boards.

We finally confirmed the total performance of TAM system as the SNR defined by the ratio of the mean value to the standard deviation of repeated measurements using the saline tank. As expected with a saline phantom, the real components of the measured data was dominant in the signal. All operating frequencies produced SNR of better than $70 \mathrm{~dB}$.

\section{Time-difference TAM images}

Figure 6(b) shows that the conductivity spectrum of agar had a smaller variation than carrot and that the conductivity of saline was relatively constant. The conductivity spectrum of carrot crossed over the conductivity spectrum of saline between $100 \mathrm{kHz}$ and $250 \mathrm{kHz}$. The contrast of agar and carrot in the saline tank was degraded at frequencies greater than $100 \mathrm{kHz}$, as expected from the real and imaginary spectra in Figures 8 and 9. Differences at frequencies less than $500 \mathrm{~Hz}$ may have been caused by the lower system performance at low frequencies as discussed previously. The contrast variation of the cylindrical carrot object showed a large difference between images and reversed above $250 \mathrm{kHz}$ in close agreement with the reference spectra in Figure 6(b). Again at less than $500 \mathrm{~Hz}$ the contrast was affected by system performance. Interestingly for the carrot object, the imaginary images showed a larger difference than the real images. These results can also be observed in the cross sections of admittance plotted through the centre of the objects in Figures 8(b),(c) and 9(b),(c). The circular object creates a larger and flatter profile particularly at $100 \mathrm{kHz}$ and $250 \mathrm{kHz}$ while the square object creates a rounder profile. This change in profiles for different shapes at many frequencies may allow us to better identify the objects in an anomaly detection algorithm.

\section{Frequency-difference TAM images}

We applied a simple frequency difference algorithm to the measurements from TAM system. Figure 10 shows the magnitude and phase images of the trans-admittance distribution obtained at 6 different frequency pairs with respect to the $500 \mathrm{~Hz}$ data. As expected the contrast of agar in the magnitude and phase images did not change significantly until the frequencies increased above $100 \mathrm{kHz}$, in agreement with the reference spectra in Figure 6(b). The contrast change of carrot in the magnitude and phase images over the frequency range also matched the reference spectra. These results show that the technique is feasible and gives us the confidence to move to tissue measurements.

In real human measurements, the spectrum will be affected by the skin-electrode interfaces since its characteristics are also varied by the operating frequency $[27,28]$. Although we have not tested this here we believe all impedance spectroscopy data will be deteriorated by this error, increasing the uncertainty of lesion detection inside the breast. An additional method to estimate the transfer function of the electrode interface is essential to remove this artefact.

Our system compares favourably with previous impedance imaging systems developed for breast cancer detection. In comparison with EIT systems, we found the significantly larger number of electrode channels enabled a resolution close to the electrode spacing of $3 \mathrm{~mm}$ while EIT systems with 72 electrodes or less have reported resolution on the centimeter scale $[29,30]$. Even with the larger number of channels, our system design allows 
a similar frame rate at 15 frames/s at $10 \mathrm{kHz}$ and covers a larger frequency range than EIT systems reporting up to 2.6 frames/s and $1 \mathrm{kHz}-1 \mathrm{MHz}$ [30]. We were able to achieve these specifications without compromising crosstalk or noise which is reflected in our average SNR values of $70 \mathrm{~dB}(5-500 \mathrm{kHz})$ and $50 \mathrm{~dB}(50 \mathrm{~Hz}-1 \mathrm{kHz})$ which is worse than that achieved in EIT systems with significantly fewer electrodes (e.g. $100 \mathrm{~dB}(<100 \mathrm{kHz})$ and $60 \mathrm{~dB}(1 \mathrm{MHz})$ [29]). The main difference here may be due to the signal level differences where EIT systems measure voltages in the $\mathrm{mV}$ range, TAM requires the measurement of currents in the nA range.

This is reflected in a similar SNR to previous high channel count admittance based systems such as the T-scan which reported a SNR of $70 \mathrm{~dB}$ at $100 \mathrm{~Hz}$ and $40 \mathrm{~dB}$ at $100 \mathrm{kHz}$ [31]. The T-scan system had a smaller frequency range and total probe size was much smaller at $72 \mathrm{~mm} \times 72 \mathrm{~mm}$ with 256 electrodes. We, and others have shown in previous work that there is depth sensitivity in impedance measurements related to the separation between electrodes. In particular Seo et al (2004) [20], have shown that the depth information and transversal position can be estimated from the measured trans-admittance maps. This estimation is based on the amount of information and so is an additional motivation for increasing the number of electrodes. This is non-invasive and practical method to find anomaly inside the breast. This technique could be improved by repositioning the array to find more direct depth information. The dual plane sensors are similar to the mammography setup as we aim to compare mammography images with impedance images for the breast in the same shape and orientation. While the EIT methods where electrodes surround the entire breast may be more sensitive to depth information, this may be a disadvantage when comparing to the mammography configuration where the breast is situated between two plates. In addition EIT images have reduced resolution in the center of the image and away from the plane of the ring of electrodes [32]. The large array of closely spaced electrodes in our TAM array does not suffer from this variation in spatial resolution. The challenge we deal with in this paper is building the instrumentation and interface to such a large number of electrodes. We demonstrate that the imaging is successful and sensitive to different shaped objects in a saline tank.

\section{Conclusions}

We found that the developed TAM system produced time-difference and frequencydifference trans-admittance images of both real and imaginary using carrot and agar objects in the saline phantom at 10 different frequencies. Contrast between background saline and anomalies changed according to the conductivity spectra of testing materials. We believe that the TAM system is capable of detecting a small anomaly in the breast from trans-admittance projection images with an average noise level of $38 \mathrm{nA}$ and $70 \mathrm{~dB}$ SNR. The large number of current-sensing electrodes significantly improves the spatial resolution. We were not able to compare to the resolution of the conventional EIT method directly. However, the resolution of TAM with this large number of electrodes is visually superior to tomographic EIT.

A TAM system is expected to be a supplementary or alternative method to detect the breast cancer. The setup of the system is similar to the X-ray mammography with the breast placed between two plates. The plates are movable to accommodate breasts with different sizes and may be rotated to provide multiple images with different projection angles. In order to quantitatively analyze the ability to detect a small 
anomaly, we will formulate a mathematical framework to describe the relationship between the admittivity of the anomaly and measured spectroscopic trans-admittance maps.

\begin{abstract}
Abbreviations
TAM: Trans-admittance mammography; ADC: Analog to digital converter; EIT: Electrical impedance tomography; TAS: Trans-admittance scanner; FR4: Fire-retardant glass laminate susbtrate; PCB: Printed circuit board; I-V: Current to voltage converter; FPGA: Field programmable gate array; DAC: Digital to analog converter; USB: Universal serial bus; SNR: Signal to noise ratio.
\end{abstract}

Competing interests

The authors declare that they have no competing interests.

\title{
Authors' contributions
}

MZ setup the system, performed and wrote up the experiments. HW developed the printed circuit boards and figures for the manuscript. AHMK wrote the system software and helped draft the manuscript. ALM assisted with analysis and gave critical revision for important intellectual content, EJW conceived the idea and revised the manuscript, and TIO designed the system and drafted the manuscript. All authors read and approved the final manuscript.

Acknowledgements

This work was supported by a grant from the Kyung Hee University in 2012 (KHU-20120821).

Received: 11 July 2012 Accepted: 5 September 2012

Published: 25 September 2012

References

1. Surowiec AJ, Stuchly SS, Barr JR, Swarup A: Dielectric properties of breast carcinoma and the surrounding tissues. IEEE Trans Biomed Eng 1998, 35:257-63.

2. Jossinet J, Schmitt M: A review of parameters for the bioelectrical characterization of breast tissue. Ann New York Acad of Sci 1999, 873:30-41.

3. Silva JEMJP, J J: Classification of breast tissue by electrical impedance spectroscopy. Med Biol Eng Comput 2000, 38:26-30

4. Hartov A, Soni N, Halter R: Breast cancer screening with electrical impedance tomography. In Electrical Impedance Tomography: Methods, History and Applications. Edited by Holder DS. Bristol, UK: IOP Publishing; 2005:167-85.

5. Holder DS: Electrical Impedance Tomography: Methods, History and Applications. Bristol, UK: IOP Publishing; 2005.

6. Soleimani M: Electrical impedance tomography system: an open access circuit design. Biomed Eng OnLine 2006, 5:28.

7. Larson-Wiseman JL: Early Breast Cancer Detection Utilizing Clustered Electrode Arrays in Impedance Imaging. PhD thesis. Troy, NY, USA: RPI; 1998.

8. Mueller JL, Isaacson D, Newell JC: A reconstruction algorithm for electrical impedance tomography data collected on rectangular electrode arrays. IEEE Trans Biomed Eng 1999, 46:1379-86.

9. Cherepenin V, Karpov A, Korjenevsky A, Kornienko V, Mazaletskaya A, Mazourov D, Meister J: A 3D electrical impedance tomography (EIT) system for breast cancer detection. Physiol Meas 2001, 22:9-18.

10. Cherepenin V, Karpov A, Korjenevsky A, Kornienko V, Kultiasov Y, Ochapkin M, O T, Meister J: Three-dimensional EIT imaging of breast tissues: system design and clinical testing. IEEE Trans Med Imag 2002, 21:662-7.

11. Kerner TE, Paulsen KD, Hartov A, Soho SK, Poplack SP: Electrical impedance spectroscopy of the breast: clinical imaging results in 26 subjects. IEEE Trans Med Imag 2002, 21:638-45.

12. Kao T, Newell JC, Saulinier GJ, Isaacson D: Distinguishability of inhomogeneities using planar electrode arrays and different patterns of applied excitation. Physiol Meas 2003, 24:403-11.

13. Kao T, Isaacson D, Newell JC, Saulnier GJ: A 3D reconstruction algorithm for EIT using a handheld probe for breast cancer detection. Physiol Meas 2006, 27:S1-11.

14. Kao T, Saulnier GJ, Xia H, Tamma C, Newell JC, Isaacson D: A compensated radiolucent electrode array for combined EIT and mammography. Physio/ Meas 2007, 28:S291-9.

15. Choi MH, Kao T, Isaacson D, Saulnier GJ, Newell JC: A reconstruction algorithm for breast cancer imaging with electrical impedance tomography in mammography geometry. IEEE Trans Biomed Eng 2007, 54:700-10.

16. Boverman G, Kao T, Kulkarni R, Kim BS, Isaacson D, Saulnier GJ, Newell JC: Robust linearized image reconstruction for multifrequency EIT of the breast. IEEE Trans Med Imag 2008, 27:1439-48.

17. Kao T, Boverman G, Kim BS, Isaacson D, Saulnier GJ, Newell JC, Choi MH, Moore RH, Kopans DB: Regional admittivity spectra with tomosynthesis images for breast cancer detection: preliminary patient study. IEEE Trans Med Imag 2008, 27:1762-8.

18. Assenheimer M, Laver-Moskovitz O, Malonek D, Manor D, Nahliel U, Nitzan R, Saad A: The T-Scan technology: electrical impedance as a disgnostic tool for breast cancer detection. Physiol Meas 2001, 22:1-8.

19. Scholz B: Towards virtual electrical breast biopsy: space-frequency MUSIC for trans-admittance data. IEEE Trans Med Imag 2002, 21:588-95.

20. Seo JK, Kwon O, Ammari H, Woo EJ: Mathematical framework and anomaly estimation algorithm for breast cancer detection: electrical impedance technique using TS2000 configuration. IEEE Trans Biomed Eng 2004, 51:1898-906.

21. Ammari H, Kwon O, Seo JK, Woo EJ: T-Scan electrical impedance imaging system for anomaly detection. SIAM J Appl Math 2004, 65:252-66. 
22. Oh TI, Lee J, Seo JK, Kim SW, Woo EJ: Feasibility of breast cancer lesion detection using a multi-frequency trans-admittance scanner (TAS) with $10 \mathrm{~Hz}$ to $500 \mathrm{kHz}$ bandwidth. Physiol Meas 2007, 28:S71-84.

23. Kim S, Lee J, Seo JK, Woo EJ, Zribi H: Multi-frequency trans-admittance scanner: mathematical framework and feasibility. SIAM J App/ Math 2008, 69:22-36.

24. Franco S: Design with Operational Amplifiers and Analog Integrated Circuits. 3rd. ed. NY, USA: McGraw-Hill; 2002.

25. Oh TI, Koo H, Lee KH, Kim SM, Lee J, Kim SW, Seo JK, Woo EJ: Validation of a multi-frequency electrical impedance tomography (mfEIT) system KHU Mark1: impedance spectroscopy and time-difference imaging. Physiol Meas 2008, 29:295-307.

26. Jun SC, Kuen J, Lee J, Woo EJ, Holder D, Seo JK: Frequency-difference EIT (fdEIT) using weighted difference and equivalent homogeneous admittivity: validation by simulation and tank experiment. Physio/ Meas 2009, 30(10):1087. [http://stacks.iop.org/0967-3334/30/i=10/a=009]

27. Oh TI, Yoon S, Kim TE, Wi H, Kim KJ, Woo EJ, Sadleir RJ: Nanofiber web textile dry electrodes for long-term biopotential recording. IEEE TBiomed Circ S doi:10.1109/TBCAS.2012.2201154. in press.

28. Woo E, Hua P, Webster J: Skin impedance measurements. Med Biol Eng Comput 1992, 30:97-102.

29. Osterman KS, Kerner TE, Williams DB, Hartov A, Poplack SP, Paulsen KD: Multifrequency electrical impedance imaging: preliminary in vivo experience in breast. Physio/ Meas 2000, 21:99.

30. Saulnier G, Liu N, Tamma C, Xia H, Kao TJ, Newell J, Isaacson D, IEEE Washington, DC: An Electrical Impedance Spectroscopy System for Breast Cancer Detection. In Engineering in Medicine and Biology Society, 2007. EMBS 2007. 29th Annual International Conference of the IEEE 2007; 2007:4154 -4157.

31. Assenheimer M, Laver-Moskovitz O, Malonek D, Manor D, Nahaliel U, Nitzan R, Saad A: The T-SCAN TM technology: electrical impedance as a diagnostic tool for breast cancer detection. Physio/ Meas 2001, 22:1.

32. Metherall P, Barber DC, Smallwood RH, Brown BH: Three-dimensional electrical impedance tomography. Nature 1996, 380(6574):509-512. http://dx.doi.org/10.1038/380509a0.

doi:10.1186/1475-925X-11-75

Cite this article as: Zhao et al:: High density trans-admittance mammography development and preliminary phantom tests. BioMedical Engineering OnLine 2012 11:75.

\section{Submit your next manuscript to BioMed Central and take full advantage of:}

- Convenient online submission

- Thorough peer review

- No space constraints or color figure charges

- Immediate publication on acceptance

- Inclusion in PubMed, CAS, Scopus and Google Scholar

- Research which is freely available for redistribution 